Atomistic simulations of material damping in amorphous silicon nanoresonators

This content has been downloaded from IOPscience. Please scroll down to see the full text. 2016 Modelling Simul. Mater. Sci. Eng. 24055015

(http://iopscience.iop.org/0965-0393/24/5/055015)

View the table of contents for this issue, or go to the journal homepage for more

Download details:

IP Address: 132.206.200.181

This content was downloaded on 13/06/2016 at $14: 39$

Please note that terms and conditions apply. 


\title{
Atomistic simulations of material damping in amorphous silicon nanoresonators
}

\author{
Sankha Mukherjee ${ }^{1}$, Jun Song ${ }^{2}$ and Srikar Vengallatore ${ }^{1}$ \\ ${ }^{1}$ Department of Mechanical Engineering, McGill University, 817 Sherbrooke Street \\ West, Montreal, QC H3A 0C3, Canada \\ 2 Department of Mining and Materials Engineering, McGill University, \\ 3610 University Street, Montreal, QC H3A 0C5, Canada \\ E-mail: srikar.vengallatore@mcgill.ca
}

Received 16 March 2016, revised 13 May 2016

Accepted for publication 24 May 2016

Published 13 June 2016

\begin{abstract}
Atomistic simulations using molecular dynamics (MD) are emerging as a valuable tool for exploring dissipation and material damping in nanomechanical resonators. In this study, we used isothermal MD to simulate the dynamics of the longitudinal-mode oscillations of an amorphous silicon nanoresonator as a function of frequency $(2 \mathrm{GHz}-50 \mathrm{GHz})$ and temperature $(15 \mathrm{~K}-300 \mathrm{~K})$. Damping was characterized by computing the loss tangent with an estimated uncertainty of $7 \%$. The dissipation spectrum displays a sharp peak at $50 \mathrm{~K}$ and a broad peak at around $160 \mathrm{~K}$. Damping is a weak function of frequency at room temperature, and the loss tangent has a remarkably high value of $\sim 0.01$. In contrast, at low temperatures $(15 \mathrm{~K})$, the loss tangent increases monotonically from $4 \times 10^{-4}$ to $4 \times 10^{-3}$ as the frequency increases from 2 $\mathrm{GHz}$ to $50 \mathrm{GHz}$. The mechanisms of dissipation are discussed.
\end{abstract}

Keywords: damping, dissipation, amorphous silicon, nanomechanical resonators, phonons

(Some figures may appear in colour only in the online journal)

\section{Introduction}

Classical molecular dynamics (MD) is emerging as a valuable technique for exploring the mechanisms of energy dissipation and predicting the magnitude of material damping (see, for instance [1-10]). The computationally-intensive nature of MD restricts the simulations to nanoscale structures (such as nanowires, nanotubes, and nanomembranes) oscillating at frequencies above $1 \mathrm{GHz}$. Although these structures are too small, and the frequencies too high, to be of interest to the current generation of commercial microelectromechanical systems (MEMS), they closely match the characteristics of devices that are being developed for a 
class of nanoelectromechanical systems (NEMS). For instance, the first report of a nanoscale device oscillating at gigahertz frequencies came in 2003 [11] and single-wall carbon nanotube devices with diameter of $2 \mathrm{~nm}$ and oscillating at frequencies as high as $280 \mathrm{GHz}$ were demonstrated in 2012 [12]. As computational techniques and NEMS technologies continue to evolve and mature, it may eventually become possible to use MD simulations to guide the design of nanomechanical resonators. This is the context and motivation for the work presented here.

Isothermal MD simulations of damping are still at an early stage of development, but several significant milestones have been achieved during the past few years. Using the NoséHoover thermostat $[13,14]$ to control temperature, damping was computed in defect-free single-crystal nickel [7] and single-crystal silicon [8,9] nanoresonators. The results indicated that dissipation is dominated by phonon damping, which is consistent with expectations based on theory and experiments [15-17]. Furthermore, the simulations correctly capture the relationship between the various measures of dissipation and dynamics [8], and the Duffing-like nonlinearity (which is commonly observed in experimental studies of NEMS [18]) emerges naturally from isothermal MD simulations $[8,9]$. Finally, the Nosé-Hoover thermostat generates thermomechanical noise in the nanomechanical device that is in quantitative agreement with the stipulations of the Equipartition Theorem and the Fluctuation-Dissipation Theorem [8].

Thus far, isothermal MD has been used to simulate resonators constructed using singlecrystal materials. Extending the technique to devices with polycrystalline and amorphous microstructures is timely and useful because such materials are widely used in NEMS technologies. It is well known that the microstructure can have a dramatic influence on damping and dissipation. In general, however, it is not yet possible to predict the magnitude, frequencydependence, and temperature-dependence of such effects from first principles [15, 16]. Therefore, dissipative phenomena in materials with complex microstructures are particularly well suited for analysis by MD. As a first step in that direction, we used isothermal MD to simulate damping in an amorphous silicon nanoresonator executing time-harmonic longitudinal oscillations at frequencies ranging from $2 \mathrm{GHz}$ to $50 \mathrm{GHz}$. The remaining sections of the paper describe the simulation methodologies and results of our study.

\section{Simulation methodology}

Isothermal MD simulations were performed using the large-scale atomic/molecular massively parallel simulator (LAMMPS) package [19]. The number of atoms $(N)$, volume $(V)$, and temperature $(T)$ were maintained constant during simulation. The temperature was controlled by coupling the structures to a thermal bath using the Nosé-Hoover thermostat, and the thermostat time constant was set to $0.01 \mathrm{ps}$ [8]. Nanoresonators composed entirely of the amorphous phase of silicon were created and simulated. The construction of the simulation cell and the amorphization of silicon are described in detail in section 2.1. The methods and protocols for computing damping are identical to those used in our previous study of singlecrystal silicon [8], and the main points are briefly summarized in section 2.2.

\subsection{Creation of amorphous silicon}

Amorphous silicon was generated by using the method of melting and quenching [20-22]. The first step was to create a fully-periodic simulation box with dimensions of $4.3 \mathrm{~nm} \times 4.3 \mathrm{~nm} \times 12.8 \mathrm{~nm}$ and consisting entirely of silicon atoms arranged in a bodycentered tetrahedral (bct) lattice with lattice parameter of $5.28 \AA$. The interaction between 
atoms was modeled using the Stillinger-Weber potential [23]. After equilibration at $1 \mathrm{~K}$, the crystalline silicon was melted by increasing the temperature to $3000 \mathrm{~K}$ over $0.05 \mathrm{~ns}$, and the liquid phase was equilibrated for $1 \mathrm{~ns}$. Subsequently, the molten Si structure was rapidly quenched from $3000 \mathrm{~K}$ to $1000 \mathrm{~K}$ at a rate of $40000 \mathrm{~K} \mathrm{~ns}^{-1}$ to generate the amorphous solid phase, and then annealed for $2 \mathrm{~ns}$. Finally, the amorphous structure was cooled to $300 \mathrm{~K}$ over $0.002 \mathrm{~ns}$, and equilibrated at $300 \mathrm{~K}$ for a further period of $200 \mathrm{~ns}$ (as a check, a series of simulations were also performed with quenching rates of $4000 \mathrm{~K} \mathrm{~ns}^{-1}$ and $2000 \mathrm{~K} \mathrm{~ns}^{-1}$ in order to rule out any artificial rate dependence on the structure and properties of the amorphous phase).

The structure was characterized using the radial distribution function (RDF), which was obtained by calculating the number of atoms within a radial distance of $r$ and $r+\mathrm{d} r$ from every other atom in the structure, and binning the results into a histogram. Figure 1 shows the RDF for amorphous Si computed using the visual molecular dynamics (VMD) program [24]. Also shown is the RDF for single-crystal silicon which exhibits multiple sharp peaks indicating long range order. The first four peaks for single-crystal Si occur at $2.38 \AA$, $3.87 \AA, 4.61 \AA$, and $5.54 \AA$. In contrast, the amorphous solid has a primary peak at $2.43 \AA$ and a second shallow peak at $3.87 \AA$, and the long range order is lost after $6 \AA$. For comparison, experimental characterization of amorphous silicon using x-ray diffraction reported the primary and second peaks at $2.36 \AA$ and $3.76 \AA[25]$.

Furthermore, we also employed bond order parameters (BOP) to characterize the amorphous phase. The BOP are dimensionless measures of geometric disorder given by

$$
q_{k}(i)=\left[\frac{4 \pi}{2 k+1} \sum_{m=-k}^{k} \frac{1}{N^{2}}\left|\sum_{j=1}^{N} Y_{k m}\left(\hat{r}_{i j}\right)\right|^{2}\right]^{0.5},
$$

where $k$ and $m$ are integers, $Y_{k m}$ are spherical harmonics, and the unit vector $\widehat{r}_{i j}$ connects atom $i$ with each of its $N$ neighboring atoms $j$ [26-29]. In the absence of any orientational order (as in an isotropic liquid), all the bond order parameters are expected to be identically zero [29]. For structures with cubic symmetry, the first three non-zero parameters are $q_{4}, q_{6}$, and $q_{8}$ [29]. Hence, these three BOPs were selected for comparing the degree of order in single-crystal and amorphous silicon. Table 1 shows the average values at $300 \mathrm{~K}$. In all cases, the BOP for the amorphous solid are significantly lower than those for the defect-free single crystal, indicating a higher level of structural disorder in the amorphous phase.

\subsection{Structural characterization}

After generating the amorphous phase of silicon, an axial fixed-free nanoresonator was defined with cross-sectional dimensions of $4.3 \mathrm{~nm} \times 4.3 \mathrm{~nm}$ and length of $L=7.6 \mathrm{~nm}$. Denoting the axial coordinate by $x$, the boundary at $x=0$ was clamped by fixing the position and velocity of two rows of atoms, as shown in figure 2. Periodic boundary conditions were imposed on all lateral surfaces, and the boundary at $x=L$ was free. The equations of motion were integrated using the velocity form of the Verlet algorithm with a time step of $1 \mathrm{fs}$.

After equilibration at $300 \mathrm{~K}$, the thermomechanical noise spectrum of the atoms at the free end was recorded and analyzed in the frequency domain to obtain a value of $284 \mathrm{GHz}$ for the fundamental frequency of longitudinal oscillations. Subsequently, static axial tests were simulated by applying a series of forces to the free end and recording the corresponding displacements. The force-displacement curve exhibits a linear response for forces less than $17 \mathrm{nN}$, corresponding to elastic axial deformation. Linear regression analysis of the elastic response gave values of $389 \mathrm{~N} \mathrm{~m}^{-1}$ for the axial stiffness and $162 \mathrm{GPa}$ for the effective Young's modulus. 


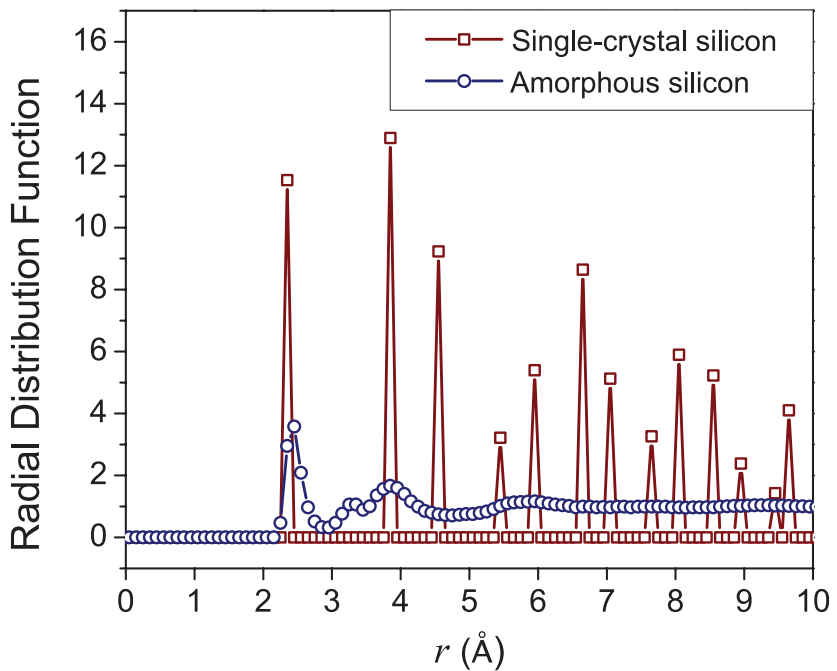

Figure 1. Molecular dynamics simulations of the radial distribution functions for single-crystal silicon and amorphous silicon at $300 \mathrm{~K}$.

Table 1. Average bond order parameters (BOP) at $300 \mathrm{~K}$.

\begin{tabular}{lll}
\hline BOP & Single-crystal Si & Amorphous Si \\
\hline$q_{4}$ & 0.772 & 0.575 \\
$q_{6}$ & 0.773 & 0.554 \\
$q_{8}$ & 0.603 & 0.552 \\
\hline
\end{tabular}

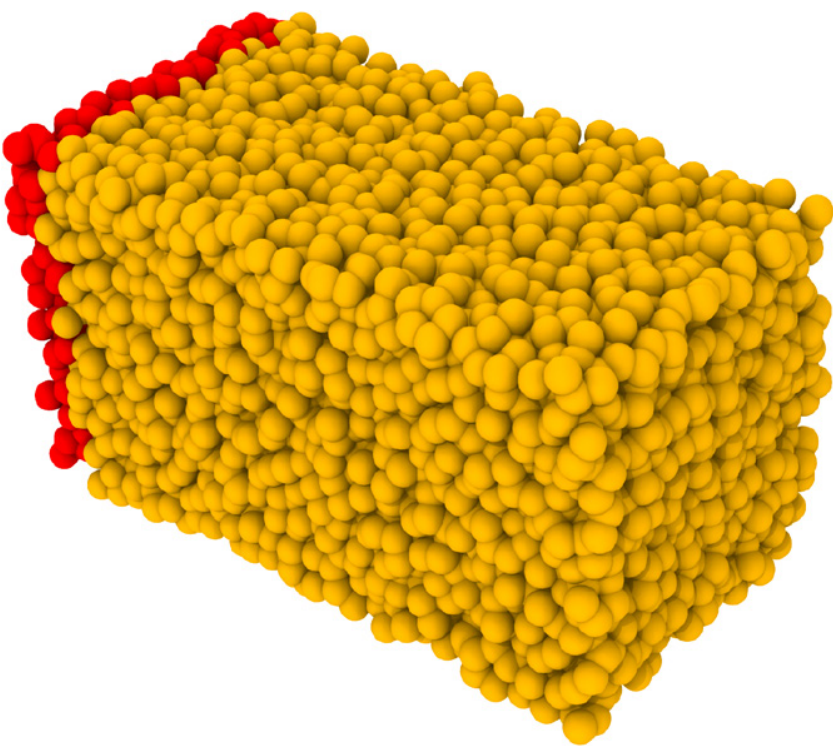

Figure 2. Atomic configuration of the amorphous silicon nanoresonator with crosssectional dimensions of $4.3 \mathrm{~nm} \times 4.3 \mathrm{~nm}$ and length of $7.6 \mathrm{~nm}$. Two layers of atoms (shown in red) are fixed in order to simulate a clamped boundary. 
The dynamics of the axial resonator was characterized in the sub-harmonic regime (that is, at frequencies lower than the fundamental natural frequency) by applying an axial timeharmonic force, $F(t)=F_{0} \sin (2 \pi f t)$, on the atoms at the free end $(x=L)$, and recording the time-series of the axial displacement. Here, $F_{0}$ is the amplitude of the force, $f$ is the frequency, and $t$ is the time. The axial response has two components: (i) spontaneous random fluctuations due to thermomechanical noise and (ii) steady-state harmonic motion. The former has a rootmean-square displacement given by $\sqrt{k_{\mathrm{B}} T / k}=3 \times 10^{-12} \mathrm{~m}$, where $k_{\mathrm{B}}$ is Boltzmann's constant and $k$ is the axial stiffness. The latter can be expressed as $X \sin (2 \pi f t-\phi)$, where $X$ is the steady-state displacement amplitude and $\phi$ is the phase angle. All simulations were performed with $F_{0}=4.3 \mathrm{nN}$ and the amplitude of the steady-state harmonic response is about five times higher than the thermal noise.

\section{Results}

Damping was quantified using the loss tangent $(\tan \phi)$. The phase angle was obtained from simulations by analyzing the harmonic force and steady-state displacement in the frequency domain. Further, in order to quantify the uncertainty in the simulations, we also computed the loss factor which is given by [8]

$$
\eta=\frac{\Delta W}{2 \pi W} .
$$

Here $W=0.5 k X^{2}$ is the maximum elastic energy during the oscillation cycle, and $\Delta W$ is the energy dissipated per unit cycle. By definition,

$$
\Delta W=\frac{1}{f \tau} \int_{0}^{\tau} F_{0} v \sin (2 \pi f t) \mathrm{d} t,
$$

where $\tau$ is the simulation time and $v$ is the axial atomic velocity [7, 8]. The loss tangent and loss factor were computed after every nanosecond. Depending on the frequency and temperature, the convergence time ranged from $100 \mathrm{~ns}$ to $300 \mathrm{~ns}$, which provides an indication of the computationally-intensive nature of the simulations. Table 2 presents the two measures of damping at $300 \mathrm{~K}$ for frequencies ranging from $2 \mathrm{GHz}$ to $50 \mathrm{GHz}$. The difference between the loss tangent and the loss factor ranges from $3.4 \%$ to $7.0 \%$. The upper bound gives an estimate of the maximum fractional uncertainty in our simulations of damping [8].

The main results of our study are presented in figure 3 in the form of graphs depicting the effects of frequency and temperature on the loss tangent. The primary conclusions are as follows.

(i) At $300 \mathrm{~K}$, damping is a weak function of frequency, and the loss tangent exhibits a remarkably high value of $\sim 0.01$.

(ii) At $15 \mathrm{~K}$, the loss tangent increases monotonically from $4 \times 10^{-4}$ to $4 \times 10^{-3}$ as the frequency increases from $0.5 \mathrm{GHz}$ to $50 \mathrm{GHz}$.

(iii) The dissipation spectrum displays a sharp internal friction peak at around $50 \mathrm{~K}$, and a broad peak at around $160 \mathrm{~K}$.

\section{Discussion}

Experimental studies of damping in amorphous silicon microresonators have been conducted only at frequencies ranging from $5.5 \mathrm{kHz}$ [30] to $13.6 \mathrm{MHz}$ [31]. Therefore, it is not yet 
Table 2. Loss tangent and loss factor for the amorphous Si nanoresonator at $300 \mathrm{~K}$.

\begin{tabular}{llll}
\hline Frequency $(\mathrm{GHz})$ & Loss tangent, $\tan \phi$ & Loss factor, $\eta$ & $\frac{\eta-\tan \phi}{\eta}(\%)$ \\
\hline 2 & $9.48 \times 10^{-3}$ & $1.02 \times 10^{-2}$ & 7.0 \\
3 & $8.86 \times 10^{-3}$ & $9.50 \times 10^{-3}$ & 6.4 \\
4 & $6.25 \times 10^{-3}$ & $6.66 \times 10^{-3}$ & 6.2 \\
5 & $7.21 \times 10^{-3}$ & $7.66 \times 10^{-3}$ & 5.8 \\
10 & $8.46 \times 10^{-3}$ & $9.05 \times 10^{-3}$ & 6.5 \\
15 & $7.41 \times 10^{-3}$ & $7.67 \times 10^{-3}$ & 3.4 \\
25 & $8.67 \times 10^{-3}$ & $9.20 \times 10^{-3}$ & 5.8 \\
30 & $8.99 \times 10^{-3}$ & $9.55 \times 10^{-3}$ & 5.8 \\
40 & $1.10 \times 10^{-2}$ & $1.17 \times 10^{-2}$ & 6.0 \\
50 & $1.19 \times 10^{-2}$ & $1.25 \times 10^{-2}$ & 4.8 \\
\hline
\end{tabular}
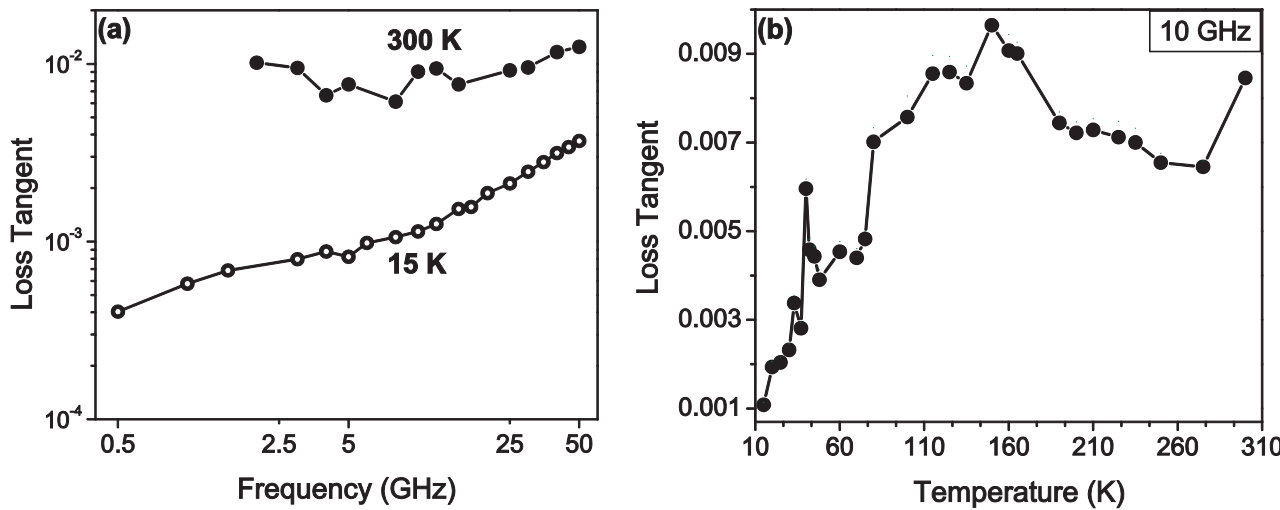

Figure 3. Results from MD simulations for the effects of (a) frequency and (b) temperature on the loss tangent for the amorphous silicon nanoresonator.

possible to compare the simulations with experimental data. Instead, we discuss our results by comparing with previous MD simulations of dissipation in single-crystal silicon nanoresonators $[8,9]$. A direct comparison can be made because both studies employed the same simulation methodology and simulation parameters (geometry, dimensions, mode and frequencies of oscillation, interatomic potential, thermostat, and integration time), as displayed in table 3. Figure 4 shows the frequency-dependence of damping for single-crystal $\mathrm{Si}$ and amorphous $\mathrm{Si}$ at $300 \mathrm{~K}$. All else being the same, damping increases by more than one order of magnitude when the microstructure changes from single crystal to amorphous. In contrast, the corresponding increase in the axial stiffness and fundamental natural frequency is only around $10 \%$. The difference in the densities of amorphous and single-crystal silicon is an artifact of the Stillinger-Weber potential [32].

\subsection{Mechanisms of dissipation}

In general, the value of the loss tangent $(\tan \phi)$ is governed by three different sources of dissipation, namely, (i) boundary damping, (ii) fluid-structure interactions, and (iii) material damping $[15,16]$. However, the first two sources are negligible in our simulations because of the 
Table 3. Comparison of simulation parameters and properties for axial nanoresonators constructed using single-crystal Si and amorphous Si.

\begin{tabular}{lll}
\hline & $\begin{array}{l}\text { Amorphous } \\
\text { silicon (current work) }\end{array}$ & $\begin{array}{l}\text { Single-crystal } \\
\text { silicon [8,9] }\end{array}$ \\
\hline Interatomic potential & Stillinger-Weber & Stillinger-Weber \\
Cross-sectional dimensions & $4.3 \mathrm{~nm} \times 4.3 \mathrm{~nm}$ & $4.3 \mathrm{~nm} \times 4.3 \mathrm{~nm}$ \\
Length & $7.6 \mathrm{~nm}$ & $7.6 \mathrm{~nm}$ \\
Thermostat & Nosé-Hoover & Nosé-Hoover \\
Temperature & $300 \mathrm{~K}$ & $300 \mathrm{~K}$ \\
Density & $2560 \mathrm{~kg} \mathrm{~m}^{-3}$ & $2315 \mathrm{~kg} \mathrm{~m}^{-3}$ \\
Speed of sound, $V_{\mathrm{s}}$ & $7955 \mathrm{~m} \mathrm{~s}^{-1}$ & $7815 \mathrm{~m} \mathrm{~s}^{-1}$ \\
Axial stiffness, $k$ & $389 \mathrm{~N} \mathrm{~m}^{-1}$ & $352 \mathrm{~N} \mathrm{~m}^{-1}$ \\
Fundamental frequency & $284 \mathrm{GHz}$ & $256 \mathrm{GHz}^{-1}$ \\
\hline
\end{tabular}

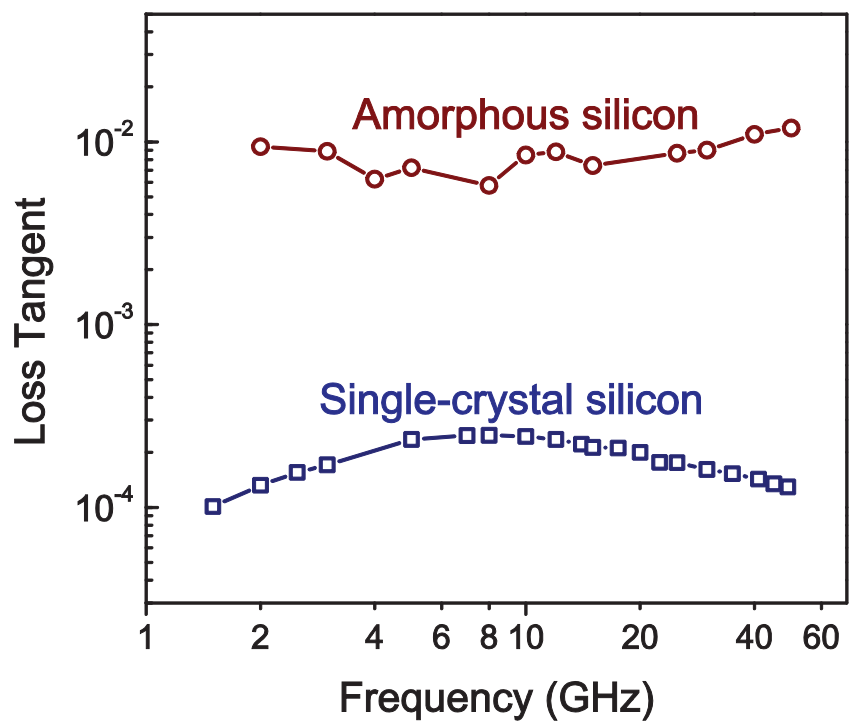

Figure 4. Comparison of results from isothermal MD simulations of damping in the longitudinal-mode oscillations of nanoresonators constructed using amorphous silicon and single-crystal silicon.

idealized boundary conditions. Therefore, the dissipation is entirely due to material damping (also called internal friction). For amorphous solids at room temperature $(300 \mathrm{~K})$, the mechanisms of material damping include quantum tunneling between two-level systems (TLS), thermally-activated relaxation of defects, thermoelastic damping, Akhiezer damping due to bulk scattering of thermal phonons, and scattering of phonons at surfaces [15, 30, 32-35]. In this section, we seek to gain some insight into the roles of these various mechanisms.

For crystalline solids, the frequency dependence of Akhiezer damping due to bulk scattering of phonons can be estimated using [15]

$$
\tan \phi_{\text {Akhiezer }}=\frac{C T \gamma^{2}}{\rho V_{\mathrm{s}}^{2}} \frac{2 \pi f \tau_{\mathrm{r}}}{1+\left(2 \pi f \tau_{\mathrm{r}}\right)^{2}} .
$$

Here, $C$ is the specific heat per unit volume, $\gamma$ is the average Grüneisen parameter, $\rho$ is the density, $V_{\mathrm{s}}$ is the speed of sound, and $\tau_{\mathrm{r}}$ is the phonon relaxation time. However, equation (4) 
cannot be applied directly to amorphous solids because of the complicated nature of thermal vibrations. Instead, we rely on the estimates obtained by Fabian and Allen [33] for sound attenuation in amorphous Si due to the scattering of thermal phonons in the Akhiezer regime. In that study, the attenuation coefficients due to Akhiezer damping $\left(\Gamma_{\text {Akhiezer }}\right)$ were computed using the Stillinger-Weber potential with atomic coordinates based on the model of Wooten, Winer and Weaire [36]. We calculated the Akhiezer loss tangent from the Fabian-Allen attenuation coefficient values according to

$$
\tan \phi_{\text {Akhiezer }}=\frac{V_{\mathrm{s}} \Gamma_{\text {Akhiezer }}}{2 \pi f},
$$

and the results are tabulated in table 4 . At $300 \mathrm{~K}$, Akhiezer damping increases monotonically from $4.59 \times 10^{-4}$ at $2 \mathrm{GHz}$ to $5.96 \times 10^{-3}$ at $50 \mathrm{GHz}$. This mechanism is insignificant at the lower end of the spectrum (contributing less than $5 \%$ of the damping at $2 \mathrm{GHz}$ ), but is responsible for over $50 \%$ of the damping observed in the nanoresonators at frequencies ranging from $25 \mathrm{GHz}$ to $50 \mathrm{GHz}$. The residual damping (that is, $\tan \phi-\tan \phi_{\text {Akhiezer }}$ ) is also presented in table 4 .

The mechanisms responsible for the residual damping are currently unknown. Comparison with experimental studies of sound attenuation in silica suggest an important role for thermally-activated relaxation of defects [34, 35]. Mousseau and Barkema [37] analyzed activated mechanisms in amorphous silicon using MD simulations and identified over 8000 processes associated with topological defects. However, correlating these processes with dissipation and quantifying the magnitude of damping are open questions of formidable difficulty.

Next, let us consider the relaxation peak at $50 \mathrm{~K}$ and $10 \mathrm{GHz}$ (figure 3(b)). Calculations of Akhiezer damping due to bulk scattering of phonons report a relaxation peak at $50 \mathrm{~K}$ and $1 \mathrm{GHz}$, but the peak vanishes above $5 \mathrm{GHz}$ [33]. Measurements of internal friction in thin films of amorphous silicon at low frequencies $(5.5 \mathrm{kHz})$ have observed relaxation peaks at temperatures ranging from $40 \mathrm{~K}$ to $60 \mathrm{~K}[30]$. The location and intensity of the peaks were found to depend on the processing conditions (hot-wire chemical vapor deposition or plasma-enhanced chemical vapor deposition) and level of hydrogenation, and the peak was attributed to structural disorder and defects in the amorphous phase [30]. However, a detailed understanding of the nature and structure of the defects has remained elusive. Thus, in common with the residual damping at $300 \mathrm{~K}$, it is possible that defect relaxation is responsible for the internal friction peak in our simulations, but further studies are required to verify this claim.

Finally, we note that our classical MD simulations cannot capture quantum phonon dynamics and tunneling between TLS. These mechanisms are negligible at room temperature, but can influence dissipation at cryogenic temperatures [15]. Therefore, ab initio methods are required for probing damping below $10 \mathrm{~K}$.

\subsection{Evaluation of the Stillinger-Weber (SW) potential}

Several interatomic potentials are available for silicon, each with its own merits and limitations [38]. We selected the Stillinger-Weber (SW) potential for our simulations because it has been extensively used, tested, and validated in simulations of the mechanical and thermodynamic properties of bulk single-crystal and amorphous silicon. However, additional simulations with other potentials are needed to evaluate whether the SW potential can capture all dissipative mechanisms, especially those associated with surface defects. Chu et al [10] compared the SW and Tersoff potentials in their study of the dissipative dynamics of singlecrystal silicon nanobeams, but the simulations were performed under non-isothermal conditions with damping arising due to energy transfer between different normal modes. Extending 
Table 4. Estimates for the contribution of Akhiezer damping to dissipation at $300 \mathrm{~K}$.

\begin{tabular}{llcl}
\hline $\begin{array}{l}\text { Frequency } \\
(\mathrm{GHz})\end{array}$ & $\begin{array}{l}\text { Akhiezer damping, } \\
\tan \phi_{\text {Akhiezer }}[33]\end{array}$ & $\frac{\tan \phi_{\text {Akhiezer }}(\%)}{\tan \phi}$ & $\begin{array}{l}\text { Residual damping, } \\
\left(\tan \phi-\tan \phi_{\text {Akhiezer }}\right.\end{array}$ \\
\hline 2 & $4.59 \times 10^{-4}$ & 4.8 & $9.02 \times 10^{-3}$ \\
3 & $6.39 \times 10^{-4}$ & 7.2 & $8.22 \times 10^{-3}$ \\
4 & $9.22 \times 10^{-4}$ & 14.8 & $5.33 \times 10^{-3}$ \\
5 & $1.17 \times 10^{-3}$ & 16.2 & $6.04 \times 10^{-3}$ \\
10 & $2.25 \times 10^{-3}$ & 26.6 & $6.21 \times 10^{-3}$ \\
15 & $3.11 \times 10^{-3}$ & 41.9 & $4.30 \times 10^{-3}$ \\
25 & $4.61 \times 10^{-3}$ & 53.1 & $4.06 \times 10^{-3}$ \\
30 & $5.02 \times 10^{-3}$ & 55.8 & $3.97 \times 10^{-3}$ \\
40 & $5.63 \times 10^{-3}$ & 51.1 & $5.37 \times 10^{-3}$ \\
50 & $5.96 \times 10^{-3}$ & 50.1 & $5.94 \times 10^{-3}$ \\
\hline
\end{tabular}

the comparison to isothermal MD simulations of damping in single-crystal and amorphous silicon is an important, but computationally-intensive, topic for further investigations.

\section{Summary}

In this paper, we used isothermal MD to simulate the dynamics of the longitudinal-mode oscillations of an amorphous silicon nanoresonator as a function of frequency $(2 \mathrm{GHz}-50 \mathrm{GHz})$ and temperature $(15 \mathrm{~K}-300 \mathrm{~K})$. Damping was characterized by computing the loss tangent with an estimated uncertainty of $7 \%$. The dissipation spectrum exhibits a sharp peak at $50 \mathrm{~K}$ and a broad peak at around $160 \mathrm{~K}$. Damping is a weak function of frequency at room temperature, and the loss tangent exhibits a remarkably high value of $\sim 0.01$. In contrast, at low temperatures $(15 \mathrm{~K})$, the loss tangent increases by an order of magnitude as the frequency increases from $2 \mathrm{GHz}$ to $50 \mathrm{GHz}$. To the best of our knowledge, this study constitutes the first application of isothermal MD to characterize dissipation in an amorphous nanoresonator. Our results present many challenging and interesting opportunities for modeling the mechanisms of dissipation that are responsible for the damping observed in the nanoresonators.

\section{Acknowledgments}

We benefited greatly from useful discussions with Z Nourmohammadi and S Joshi, and from many constructive comments and suggestions from an anonymous referee. This study was performed using resources provided by Compute Canada. Financial support from the Natural Sciences and Engineering Research Council of Canada (NSERC) is gratefully acknowledged.

\section{References}

[1] Rudd R E and Broughton J Q 1999 Atomistic simulation of MEMS resonators through the coupling of length scales J. Model. Simul. Microsyst. 129-38

[2] Midtvedt D, Croy A, Isacsson A, Qi Z and Park H S 2014 Fermi-Pasta-Ulam physics with nanomechanical graphene resonators: intrinsic relaxation and thermalization from flexural mode coupling Phys. Rev. Lett. 112145503 
[3] Guo W, Guo Y, Gao H, Zheng Q and Zhong W 2003 Energy dissipation in gigahertz oscillators from multiwalled carbon nanotubes Phys. Rev. Lett. 91125501

[4] Vallabhaneni A K, Ruan X, Rhoads J F and Murthy J 2012 A band-pass filter approach within molecular dynamics for the prediction of intrinsic quality factors of nanoresonators $J$. Appl. Phys. 112074301

[5] Zhan H F and Gu Y T 2012 A fundamental numerical and theoretical study for the vibrational properties of nanowires J. Appl. Phys. 111124303

[6] Jiang H, Yu M F, Liu B and Huang Y 2004 Intrinsic energy loss mechanisms in a cantilevered carbon nanotube beam oscillator Phys. Rev. Lett. 93185501

[7] Kunal K and Aluru N R 2011 Akhiezer damping in nanostructures Phys. Rev. B 84245450

[8] Nourmohammadi Z, Mukherjee S, Joshi S, Song J and Vengallatore S 2015 Methods for atomistic simulations of linear and nonlinear damping in nanomechanical resonators J. Microelectromech. Syst. 24 1462-70

[9] Mukherjee S 2016 Molecular dynamics simulations of material damping and nonlinear oscillations in nanoresonators $P h D$ Thesis McGill University

[10] Chu M, Rudd R E and Blencowe M P 2007 The role of reconstructed surfaces in the intrinsic dissipative dynamics of silicon nanoresonators (arXiv: 0705.0015v1 [cond-mat.mtrl-sci])

[11] Huang X M H, Zorman C A, Mehregany M and Roukes M L 2003 Nanodevice motion at microwave frequencies Nature $\mathbf{4 2 1} 496$

[12] Island J O, Tayari V, McRae A C and Champagne A R 2012 Few-hundred GHz carbon nanotube nanoelectromechanical systems (NEMS) Nano Lett. 12 4564-9

[13] Hoover W G 1985 Canonical dynamics: equilibrium phase-space distributions Phys. Rev. A 31 1695-7

[14] Nosé S 1984 A unified formulation of the constant temperature molecular-dynamics methods J. Chem. Phys. 81 511-9

[15] Braginsky V B, Mitrofanov V P and Panov V I 1985 Systems with Small Dissipation (Chicago, IL: University of Chicago Press)

[16] Joshi S, Hung S and Vengallatore S 2014 Design strategies for controlling damping in micromechanical and nanomechanical resonators EPJ Tech. Instrum. 15

[17] Ghaffari S, Chandorkar S A, Wang S, Ng E J, Ahn C H, Hong V, Yang Y and Kenny T W 2013 Quantum limit of quality factor in silicon micro and nano mechanical resonators Sci. Rep. 33244

[18] Postma H W C, Kozinksy I, Husain A and Roukes M L 2005 Dynamic range of nanotube and nanowire-based electromechanical systems Appl. Phys. Lett. 86223105

[19] Plimpton S 1995 Fast parallel algorithms for short-range molecular dynamics J. Comput. Phys. $1171-19$

[20] Luedtke W D and Landman U 1989 Preparation, structure, dynamics, and energetics of amorphous silicon: a molecular-dynamics study Phys. Rev. B 40 1164-74

[21] Vollmayr K, Kob W and Binder K 1996 Cooling-rate effects in amorphous silica: a computersimulation study Phys. Rev. B 54 15808-27

[22] Argon A S and Demkowicz M J 2006 Atomistic simulation and analysis of plasticity in amorphous silicon Phil. Mag. 86 4153-72

[23] Stillinger F H and Weber T A 1985 Computer simulation of local order in condensed phases of silicon Phys. Rev. B 31 5262-71

[24] Humphrey W, Dalke A and Schulten K 1996 VMD: visual molecular dynamics J Mol. Graph. $1433-8$

[25] Laaziri K, Kycia S, Roorda S, Chicoine M, Robertson J L, Wang J and Moss S C 1999 High resolution radial distribution function of pure amorphous silicon Phys. Rev. Lett. 82 3460-3

[26] Steinhardt P J, Nelson D R and Ronchetti M 1983 Bond-orientational order in liquids and glasses Phys. Rev. B 28 784-805

[27] Song J and Srolovitz D J 2007 Atomistic simulation of multicycle asperity contact Acta Mater. 55 4759-68

[28] Kim T H, Goldman A I and Kelton K F 2008 Structural study of supercooled liquid silicon Phil. Mag. 88 171-9

[29] Steinhardt P J, Nelson D R and Ronchetti M 1981 Icosahedral bond orientational order in supercooled liquids Phys. Rev. Lett. 47 1297-300

[30] Liu X, Spiel C L, Merithew R D, Pohl R O, Nelson B P, Wang Q and Crandall R S 2006 Internal friction of amorphous and nanocrystalline silicon at low temperatures Mater. Sci. Eng. A 442 307-13 
[31] Gualdino A, Gaspar J, Chu V and Conde J P 2015 Sub-micron gap in-plane micromechanical resonators based on low-temperature amorphous silicon thin-films on glass substrates J. Micromech. Microeng. 25075026

[32] Biswas R, Grest G S and Soukoulis C M 1987 Generation of amorphous-silicon structures with use of molecular dynamics simulations Phys. Rev. B 36 7437-41

[33] Fabian J and Allen P B 1999 Theory of sound attenuation in glasses: the role of thermal vibrations Phys. Rev. Lett. 82 1478-81

[34] Rat E, Foret M, Massiera G, Vialla R, Arai M, Vacher R and Courtens E 2005 Anharmonic versus relaxational sound damping in glasses. I. Brillouin scattering from densified silica Phys. Rev. B 72214204

[35] Vacher R, Courtens E and Foret M 2005 Anharmonic versus relaxational sound damping in glasses. II. Vitreous silica Phys. Rev. B 72214205

[36] Wooten F, Winer K and Weaire D 1985 Computer generation of structural models of amorphous Si and Ge Phys. Rev. Lett. 54 1392-5

[37] Mousseau N and Barkema G T 2000 Activated mechanisms in amorphous silicon: an activationrelaxation-technique study Phys. Rev. B 61 1898-906

[38] Balamane H, Halicioglu T and Tiller W A 1992 Comparative study of silicon empirical interatomic potentials Phys. Rev. B 46 2250-79 